\title{
Adaptive Population Sizing Genetic Algorithm Assisted Maximum Likelihood Detection of OFDM Symbols in the Presence of Nonlinear Distortions
}

\author{
K. Seshadri Sastry, M.S.Prasad Babu \\ Associate Professor,Department of AE\&IEGandhi Institute of Engineering and Technology,Gunupur, Odisha,India \\ Professor,Department of CS\&SE,Andhra University,Visakhapatnam,India. \\ aditya_shas@yahoo.com,drmsprasadbabu@yahoo.co.in
}

\begin{abstract}
This paper presents Adaptive Population Sizing Genetic Algorithm (AGA) assisted Maximum Likelihood (ML) estimation of Orthogonal Frequency Division Multiplexing (OFDM) symbols in the presence of Nonlinear Distortions. The proposed algorithm is simulated in MATLAB and compared with existing estimation algorithms such as iterative DAR, decision feedback clipping removal, iteration decoder, Genetic Algorithm (GA) assisted ML estimation and theoretical ML estimation. Simulation results proved that the performance of the proposed AGA assisted ML estimation algorithm is superior compared with the existing estimation algorithms. Further the computational complexity of GA assisted ML estimation increases with increase in number of generations or/and size of population, in the proposed AGA assisted ML estimation algorithm the population size is adaptive and depends on the best fitness. The population size in GA assisted ML estimation is fixed and sufficiently higher size of population is taken to ensure good performance of the algorithm but in proposed AGA assisted ML estimation algorithm the size of population changes as per requirement in an adaptive manner thus reducing the complexity of the algorithm.
\end{abstract}

Index Terms - Genetic Algorithm, ML estimation, OFDM

\section{INTRODUCTION}

OFDM represents an efficient technique distinguished for high-speed digital transmission over multipath fading channels; the main advantage of OFDM is its multipath delay spread tolerance and efficient spectral usage by allowing overlapping in the frequency domain. OFDM has emerged as a popular technique, in the WLAN domain, such as the IEEE 802.11a and the HIPERLAN/2, in broadcasting domain, such as the Digital Video Broadcasting for Terrestrial Television (DVB-T) and the Terrestrial Digital Multimedia/Television Broadcasting DMB-T [1]. But OFDM-based multi-carrier systems are much more sensitive to the nonlinear distortion [2] and channel-estimation errors occurring in time-varying multipath fading channels [3]. In this paper, we are focusing on the problem of the optimum OFDM symbol recovery in the presence of nonlinear distortions.

In wireless network deployment, tight requirements of power efficiency impose the use of saturating radio frequency (RF) power amplifiers. It is shown in [4], [5], and [6] that a saturating nonlinearity produces a sort of self-interference (clipping noise) which depends on the transmitted symbols and the in out characteristics of nonlinear blocks. Many researchers have proposed various algorithms in order to reduce the adverse effect of clipping noise on OFDM systems. All such algorithms are suboptimum or quasi-optimum. In [7] method authors proposed OFDM symbol recovery method which is based on the recursive application of Bayesian inference. The proposed algorithm is characterized by a consistently increased computational complexity, but results in terms of better in severe clipping conditions. In [8] and [9] methods to reduce the effects of clipping on OFDM signals are proposed. In [5] and [6], decision-directed iterative algorithms were proposed to sharply reduce the computational complexity of OFDM receivers to a polynomial order with respect to the subcarrier number. Such methodologies are very attractive from a computational point of view, but they are not effective for severe clipping.

The advent of evolutionary computation has inspired new resources for optimization problem solving, such as the optimal design of code division multiple access (CDMA) and fuzzy system. In contrast to traditional computation systems which may be good at accurate and exact computation but have brittle operations, evolutionary computation provides a more robust and efficient approach for solving complex real world problem. Many evolutionary algorithms, such as Genetic algorithm (GA) [10,11,12,13,14,15], ant colony optimization (ACO) [1], simulated annealing (SA) [16] and particle swarm optimization (PSO) $[16,17,18,19,19,20]$, have been proposed. GAs is a stochastic search procedure based on the mechanics of natural selection, genetics, and evolution. Since these methods simultaneously evaluate many points in the 
search space, they are more likely to find the global solution of a given problem. In genetic algorithms the degree of convergence depends on the number of generations $G$ and/or the population size $P$, but the complexity of genetic algorithms increases with increase in size of population and/or number of generations. In [21] authors listed various adaptive population sizing schemes in genetic algorithms

Theoretical ML detection cannot be adopted in realworld applications, and suboptimum detection strategies should be investigated. A feasible solution has been proposed by Tellado et al. in [5]. The decision-aided reconstruction (DAR) iterative approach shown in [6],[22]. The suboptimum iterative ML detection proposed in [31] initially considers the vector of bit decision provided by the conventional OFDM demodulator. In [23] authors presented genetic algorithm assisted ML detection which performs better than iterative methods at the cost of high computational complexity. In this paper efforts are made to reduce the computational complexity of [23]. We present Adaptive Population Sizing Genetic Algorithm assisted Maximum Likelihood estimation with superior performance (compared to [5],[6],[22], [31] and [23]) and low complexity (compared to [31] and [23]).

\section{SYSTEM MODEL}

The analytical expression for a generic multicarrier OFDM symbol transmitted during the generic signaling interval of duration $\mathrm{T}$ is given as follows

$$
S_{i}(t)=A_{s} \sum_{k=0}^{N-1} s_{k, i} \exp \left(\frac{j 2 \pi k t}{T}\right) \Pi(t-i T)
$$

Where $A_{s}$ is the carrier amplitude, $s_{i} \stackrel{\Delta}{=}\left\{s_{k, i}, k=0, \ldots, N-1\right\}$ is the vector of the M -level complex symbols transmitted over the $\mathrm{N}$ subcarriers (where $\mathrm{M}$ is the level of modulation), $\Pi(t)$ is the rectangular waveform of unit amplitude. Applying IFFT to $S_{i}$ would result in $w_{n}$ which could be given as

$$
w_{n, i}=A_{s} \sum_{k=0}^{N-1} s_{k, i} \exp \left(\frac{j 2 \pi k t}{T}\right), n=0,1,2 . .,(N-1)
$$

Presence of solid-state power amplifier (SSPA) in the circuit introduces an amplitude distortion which can be implemented by introducing a nonlinear memoriless block whose mathematical expression is given below (normalized Rapp model [32])

$$
g(x)=\frac{\mid x}{\left\{1+\left(\frac{x}{\alpha}\right)^{1 / 2 \beta}\right\}} x \in C \quad \alpha, \beta \in \mathfrak{R}
$$

The amount of distortion can be measured (in decibels) in terms of clip level (CL), defined as $C L \stackrel{\Delta}{=} 20 \log _{10}\left(\alpha / \sigma_{x}\right)$, where $\sigma_{x}{ }^{2}$ is the variance of input signal. The output from the nonlinear memoriless block is given by [4]

$$
\begin{aligned}
& w_{n, i}^{D} \stackrel{\Delta}{=} g\left(w_{n, i}\right)=\lambda^{g} w_{n, i}+\Omega_{n}\left(s_{i}, g\right), \\
& n=0,1, \ldots \ldots,(N-1)
\end{aligned}
$$

Where $\mathrm{g}($.$) is the nonlinear distortion function, and \lambda^{g}$ is a constant chosen in order to minimize the mean square error (MSE). It was shown in [2] for a wide range of meaningful $\mathrm{CL}$ values, the approximation $\lambda^{g} \cong 1$ holds good which allows us to rewrite the above equation as

$$
w_{n, i}^{D}=w_{n, i}+\Omega_{n}\left(s_{i}, g\right) \quad n=0,1 \ldots,(N-1)
$$

At the receiver the OFDM demodulator performs an FFT over the received baseband discrete-time sequence and provides the following output

$$
r_{k, i}^{D}=s_{k, i}+\Phi_{k}\left(s_{i}, g\right), k=0,1,2, \ldots,(N-1)
$$

Where $\Phi_{k}\left(s_{i}, g\right)=F F T\left(\Omega_{N}\left(s_{i}, g\right)\right)$ is the $\mathrm{k}^{\text {th }}$ sample of the out-of-band distortion sequence or clipping noise, resulting at the output of the OFDM demodulator [2].

\section{PROPOSED ADAPTIVE GENETIC ALGORITHM BASED ML ESTIMATION}

ML estimation is the optimum symbol estimation in the presence of nonlinear distortion and additive Gaussian noise which would minimize the equation below by minimizing $\hat{S}_{i}$

$\Lambda\left(\hat{s}_{i}\right)=\left\|y_{i}-\left[\hat{s}_{i}+\Phi\left(\hat{s}_{i}, g\right)\right]\right\|^{2}$

Where $y_{i}=r_{i}^{D}+\eta$ is the received signal sample vector and $\eta$ is Additive white Gaussian Noise (AWGN) added in the channel, The nonlinear distortion is deterministic and completely known; therefore, the MLbased computation of $\hat{S}_{i}$ is theoretically possible, but the computational load grows exponentially with $\mathrm{N}$ (number of subcarriers used in OFDM system) which may range from 64 (e.g., HYPERLAN 2 system) to 2048 (VDSLDMT standard). To decrease the computational complexity we propose adaptive population sizing genetic algorithm for ML estimation of $\hat{s}_{i}$.

Genetic Algorithm (GA) is a famous probabilistic search technique based on the principles of biological 
evolution. Similar to biological organism which evolves to adapt to its environment, Genetic Algorithm follows a path of analysis from which a design evolves, one that is optimal for the environmental restrictions placed upon it. Genetic Algorithm uses probabilistic transition rules to select someone to reproduce or to die so as to guide their search toward regions of the search space with likely improvement. Thus, Genetic Algorithm is a powerful and globally stochastic search and optimization technique and is widely used in optimization problems.

First, we define the trial data vector in our proposed Genetic Algorithm based on ML estimator as $\hat{b}=\left[\hat{b_{1}}, \hat{b_{2}}, \ldots \hat{b_{k}}\right]$ for $\mathrm{N}$ subcarriers then

$$
\hat{s}_{i}=\sum_{k=1}^{N} \sqrt{E_{k}} \hat{b}_{k}
$$

Where $E_{k}$ is signal energy per bit of $k^{\text {th }}$ user, $b_{k}$ is the $k^{\text {th }}$ user's data modulated by binary phase shift keying (BPSK),. Fig. 1 shows a flow chart of the proposed Genetic Algorithm assisted ML detection. The proposed Genetic Algorithm based ML detection will return optimum or near-optimum estimated value $\hat{b}_{k}$ of $\hat{s}_{i}$ for the $k^{\text {th }}$ user.

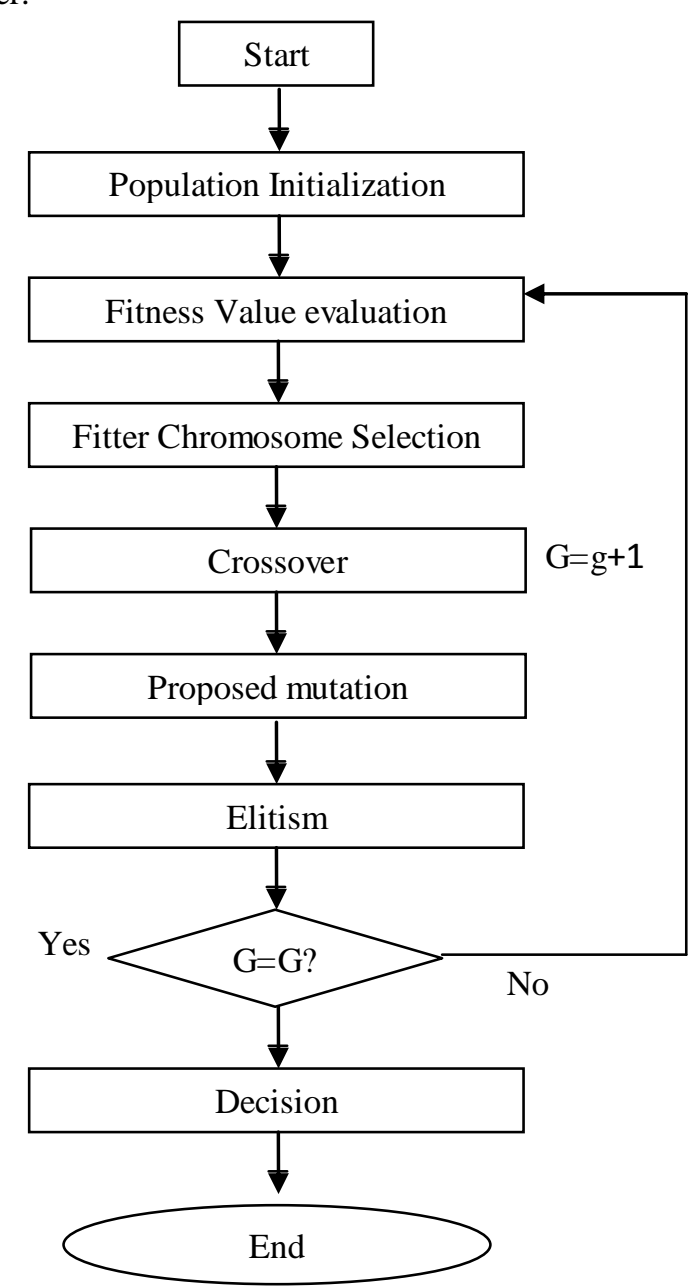

Figure 1 Flow chart of the proposed Genetic Algorithm assisted ML detection scheme
The steps involved in implementing ML detection using genetic algorithm is described below.

\section{Start with encoding}

To start Genetic Algorithm, the trial data vector $\hat{b}$ must first be encoded into binary string form. The encoded binary string is regarded as a chromosome in Genetic Algorithm and its elements are regarded as genes BPSK modulation has already been employed in the transmission model. Thus, the chromosome encoding procedure is unnecessary. The number of genes in a chromosome, which is the number of bits in a trial data vector for BPSK, is the number of users. The number of bits in a symbol is equal to the number of bits in a gene.

\section{Population initialization}

After encoding, an initial population consisting of $P$ members, called individuals, is created. In our proposed Genetic Algorithm, each individual or chromosome in the population is represented by a vector of $K$ bits. In this vector, each bit is a trial data belonging to one of the $K$ users. The $p^{\text {th }}$ individual which is the estimated value of $b=\left[b 1, b 2, \ldots ., b_{k}\right]^{T}$. After initialization of the population of $P$ individuals the optimization process starts, in which the initial generation $g$ is 1 .

\section{Fitness evaluation}

In the procedure of this fitness evaluation, Genetic Algorithm exploits an objective function (OF) to evaluate the fitness of each individual in the population, which represents how closely each individual matches the optimum individual. The optimum individual can maximize the objective function value. We will now discuss how to find the objective function. The solution of optimum detection is the most likely trial data vector $\hat{b}_{\text {opt }}$ that minimizes the value of $\Lambda\left(\hat{s}_{i}\right)$.

\section{Selection}

To evolve the population, some excellent individuals will be chosen to constitute a future population for reproduction. The fitter individuals with better genes are more likely to be selected to produce the descendant individuals. So, the rule of selection is based on their fitness function values.

\section{Crossover}

Crossover is the operation by which the selected individuals exchange their genes to produce pairs of descendants. The crossover operation randomly chooses one cutting point or many cutting points and exchanges the binary strings of individuals before or after the cutting points. For example, after a cut in the first bit in each of the two strings 0011 and 1010, these two strings are crossed over to produce a new pair of descendants 0010 and 1011. Since the offspring inherit the merits of their parents, they are expected to be superior to their parents.

\section{Mutation}


Mutation is the proposed complementary error function to increase the diversity of the population, this mutation operation randomly changes some of the crossover result genes. Without mutation, the GA's search falls into local optima. Thus, the mutation operation is crucial to the success of Genetic Algorithm. A mutation probability $p_{m}^{(i, j)}$ which is relative to a signal-to-noise ratio (SNR) from $i$ to $j$ is defined. It can be calculated with the help of a complementary error function which can be given by.

$$
\operatorname{erfc}(x)=\frac{2}{\sqrt{\pi}} \int_{x}^{\infty} e^{-t^{2}} d t, x \geq 0
$$

\section{Elitism}

An elitism operation is used to avoid losing excellent individuals which have higher fitness or greater $\mathrm{OF}$ values from one generation to another. The operation copies a small part of the best parent individuals, and replaces the worst offspring.

\subsection{Adaptive Population Sizing Genetic Algorithm}

Genetic Algorithm is convergent, but the degree of convergence depends on the number of generations $G$ and/or the population size $P[8]$. As stated above, if $P$ and/or $G$ are sufficiently large, our proposed Genetic Algorithm based ML Detection approaches the optimum maximum likelihood detection results. However, increasing $P$ increases the computational complexity of the ML Detection system. It is impractical to pursue a slightly lower Bit Error Rate performance at the expense of much more computational complexity. So, we employ adaptive population sizing genetic algorithm to solve the complexity problem. Besides the reduction of computational complexity, the proposed adaptive population sizing genetic algorithm can improves BER performance.

In [1] Population Resizing based on Fitness Improvement Genetic Algorithm was proposed. The proposed algorithm is similar to a traditional Genetic Algorithm but at the end of the typical selection, reproduction, and mutation steps, the population size can grow or shrink based on an improvement of the best fitness contained in the population.
The population size changes when there is an improvement in best fitness, or when there is no improvement in the best fitness for a "long time". If neither of the above occurs, the population size shrinks by a small percentage (1-5\%). In their work, the growth rate $X$ for a population is given by,

$$
\begin{aligned}
& X=\text { incFactor } \times(\text { MaxmEvalNum }- \text { CurrEvalNum }) \\
& \times \frac{\max \text { mFitness }_{\text {new }}-\max \text { mFitness }_{\text {old }}}{\text { ini } \max \text { fitness }}
\end{aligned}
$$

where incFactor is a parameter in the interval $(0,1)$, MaxmEvalNum is the maximum number of fitness evaluations allowed for the whole run currentEvalNum is the current evaluation number, and maxmFitness $s_{\text {new }}$, maxmFitness $_{\text {old }}$, and inimaxfitness, are the best fitness values in the current, previous, and initial generation. The initial population size, as well as a minimum and maximum population sizes in which the algorithm must operate should be specified.

\section{EXPERIMENTAL RESULTS}

In order to estimate the performance of the proposed Adaptive population sized Genetic Algorithm based ML estimator, an OFDM system was simulated using MATLAB with a bit rate of $4 \mathrm{Mb} / \mathrm{s}$ and number of subcarriers $\mathrm{N}$ equal to $64,16 \mathrm{QAM}$ as modulation scheme and $2 / 3$ convolutional coding. Fig 2 gives comparison of BER results versus SNR with clip level $(C L)=5 \mathrm{db}$ between proposed adaptive population sizing GA (AGA) assisted ML estimation, conventional GA assisted ML estimation[5], estimation provided by iterative DAR[3], decision feedback clipping removal [4] and iteration decoder [2] symbol-estimation algorithms. As far as the parameterization of the adaptive population sized Genetic Algorithm (AGA) is concerned, we selected initialPopSize as 120 , increaseFactor as $0.2, \mathrm{~V}$ as 500, decreaseFactor as 0.5, minPopSize as 20 and maxPopSize as 100. For conventional genetic algorithm initial population size is taken as 200 and number of generations taken are 250 . 


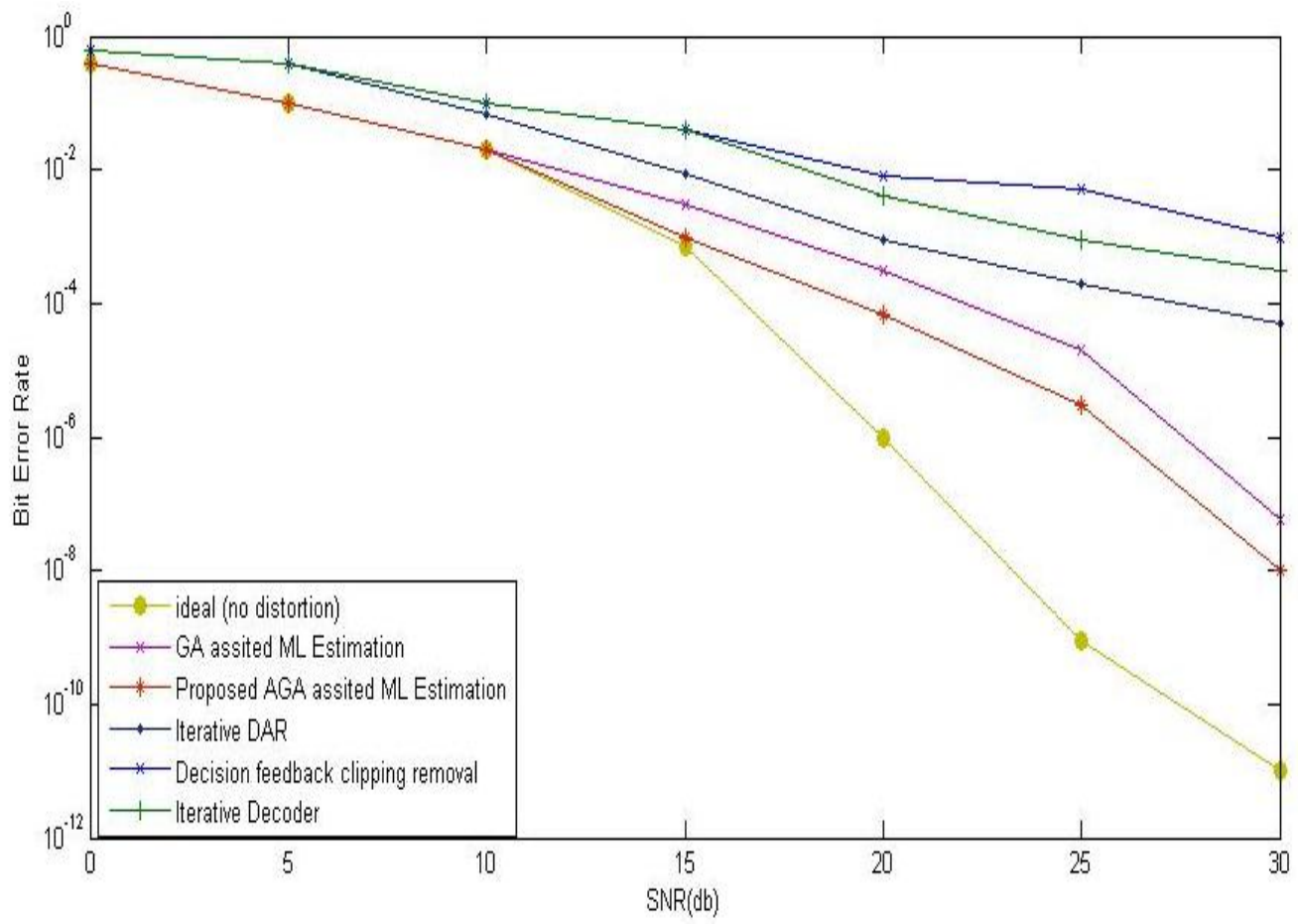

Figure 2. Comparison of BER results versus SNR between proposed adaptive population sizing GA ML estimation, conventional GA assisted ML estimation and estimation provided by the different symbol-estimation algorithms

As shown in fig 2 the decision feedback clipping removal based ML detection exhibits a severe error floor. On the other hand, iterative DAR performed better than iterative decoder, thus confirming its improved robustness for low CL values. GA assisted ML estimation algorithm shown better performance than the iterative approaches and decision feedback clipping removal based ML detection methods. But the proposed AGA assisted ML estimation provides much better results than all state-of-the-art algorithms used for comparison, with a dramatic BER decrease for high SNRs. Table 1 shows the comparison of computational complexity of the proposed adaptive population sizing GA (AGA) assisted ML estimation, conventional GA assisted ML estimation and estimation provided by iterative DAR, decision feedback clipping removal, iteration decoder symbol-estimation algorithms and theoretical ML estimation method. The computational complexity of GA assisted ML estimation increases with increase in number of generations or/and size of population, in the proposed AGA assisted ML estimation algorithm the population size is adaptive and depends on fitness function given in equation (10). The population size in GA assisted ML estimation is fixed and sufficiently higher size of population is taken to ensure the performance of algorithm but in proposed AGA assisted ML estimation algorithm the size of population is taken as per requirement in an adaptive manner thus reducing the complexity of the algorithm. Even though the computational complexity of proposed AGA assisted ML estimation algorithm is more compared to decision feedback clipping removal algorithm, Iterative DAR algorithm and Iterative decoder algorithm, its BER (Bit Error Rate) performance shown in fig 2 is much better, further the computational complexity of the proposed AGA assisted ML estimation is less compared to existing GA assisted ML estimation and theoretical ML estimation. Considering the results shown in fig 2 and table 1 it is confirmed that the performance of the proposed AGA assisted ML estimation algorithm is superior to existing ML estimation algorithms.

\section{CONCLUSION}

This paper presents Adaptive Population Sizing Genetic Algorithm (AGA) assisted Maximum Likelihood (ML) estimation of Orthogonal Frequency Division Multiplexing (OFDM) symbols in the presence of Nonlinear Distortions. The proposed detector is simulated in MATLAB and compared with existing estimation algorithms such as iterative DAR, decision feedback clipping removal, iteration decoder, Genetic Algorithm (GA) assisted ML estimation and theoretical

Table 1 Comparison of computational complexity of the proposed adaptive population sizing GA ML estimation, conventional GA assisted ML estimation and estimation provided by the different symbol-estimation algorithms

\begin{tabular}{|l|l|l|}
\hline Estimation Algorithm & $\begin{array}{l}\text { Order of Computational } \\
\text { Complexity }\end{array}$ & perations per Data symbol ( $\mathrm{N}=64)$ \\
\hline
\end{tabular}




\begin{tabular}{|l|l|l|}
\hline $\begin{array}{l}\text { Proposed AGA assisted ML } \\
\text { estimation }\end{array}$ & $\begin{array}{l}\left(P_{C}+P_{M}\right) \delta_{\text {gen }} \Gamma_{\text {size }} \\
\delta_{\text {gen }}=250, \Gamma_{\text {size }}=\text { Adaptive }\end{array}$ & $150-320$ \\
\hline $\begin{array}{l}\text { GA assisted ML estimation } \\
{[5]}\end{array}$ & $\left(P_{\text {gen }}+P_{M}\right) \delta_{\text {gen }} \Gamma_{\text {size }}$ & $\left.640, \Gamma_{\text {size }}=200\right)$ \\
\hline $\begin{array}{l}\text { Decision feedback clipping } \\
\text { removal[4] }\end{array}$ & $\left(N+2 N \log _{2} N\right) \chi(\chi=3)$ & 39 \\
\hline $\begin{array}{l}\text { Iterative DAR[3] } \\
\text { Iterative decoder[2] }\end{array}$ & $\left(2 N+2 N \log _{2} N\right) \chi$ & 56 \\
\hline Theoretical ML Estimation & $\left(N+2 N \log _{2} N\right) \chi$ & 39 \\
\hline
\end{tabular}

ML estimation. The proposed AGA assisted ML estimation provides much better results than all state-ofthe-art algorithms used for comparison, with a dramatic BER decrease for high SNRs. Even though the computational complexity of proposed AGA assisted ML estimation algorithm is more compared to Decision feedback clipping removal, Iterative DAR and Iterative decoder its BER (Bit Error Rate) performance shown in fig 2 is much better, further the computational complexity of the proposed AGA assisted ML estimation is less compared to existing GA assisted ML estimation and theoretical ML estimation. Considering the results shown in fig 2 and table 1 it is confirmed that the performance of the proposed AGA assisted ML estimation algorithm is superior to existing ML estimation algorithms.

\section{REFERENCES}

[1] Z. W. Zheng, Z. X. Yang, C. Y. Pan, and Y. S. Zhu, "Cutoff Rate andOutage Probability Performance Comparisons Between DVB-T andDMB-T Systems Under Mobile Multipath Channels," IEEE Trans.Broadcasting, Vol. 49, No. 4, 2003, pp. 390397. DOI: $10.1109 /$ TBC.2003.819524

[2] IEEE Std. 802.11a-1999, "Wireless LAN MAC and PHY Specifications High-Speed Physical Layer in the $5 \mathrm{GHz}$ Band," ISO/IEC 8802-11:1999 (E) / Amd 1: 2000 (E), New York: IEEE, 2000.

[3] ETSI, "Broadband radio access networks (BRAN); HIPERLAN Type 2; Physical (PHY) layer”, ETSI TS 101475, v1.1.1, April 2000.
[4] E. Costa, M. Midrio, and S. Pupolin, "Impact of nonlinearities on OFDM transmission system performance," IEEE Commun. Lett., vol. 3, no. 2, pp. 37-39, Feb. 1999. DOI: 10.1109/4234.749355

[5] J. Tellado, L. Loo, and J. Cioffi, "Maximumlikelihood detection of nonlinearly distorted multicarrier symbols by iterative decoding," IEEE Trans. Commun., vol. 51, no. 2, pp. 218-227, Feb. 2003. DOI:10.1109/TCOMM.2003.809289

[6] D. Kim and G. L. Stuber, "Clipping noise mitigation for OFDM by decision-aided reconstruction," IEEE Commun. Lett., vol. 3, no. 1, pp. 4-6, Jan. 1999. DOI: $10.1109 / 4234.740112$

[7] D. Declercq and G. B. Giannakis, "Recovering clipped OFDM symbols with Bayesian inference," in Proc. IEEE Signal Process. Conf., Istanbul, Turkey, Jun. 2000, vol. 1, pp. 157-160. DOI: $10.1109 /$ ICASSP.2000.861898

[8] X. Li and L. J. Cimini, "Effects of clipping and filtering on the performance of OFDM," IEEE Commun. Lett., vol. 2, no. 5, pp. 131-133,May 1998. DOI:10.1109/4234.673657

[9] R. Dinis and A. Gusmão, “A class of nonlinear signalprocessing schemes for bandwidth-efficient OFDM transmission with low envelope fluctuations," IEEE Trans. Commun., vol. 52, no. 11, pp.2009-2018, Nov. 2004. DOI:10.1109/TCOMM.2004.836567

[10] Abedi, S., \& Tafazoll, R. (2002). Genetically modified multiuser detection for code division multiple access systems. IEEE Journal on Selected 
Areas in Communications, 20, 463-473,. DOI:10.1109/49.983373

[11]. Ergun, C.,\&Haciglu, K. (2000).Multiuser detection using a genetic algorithm in CDMAcommunication systems. IEEE Transactions on Communications, 48, 1374-1383. DOI: $10.1109 / 26.864174$

[12]. Goldberg, D. E. (1989). Genetic algorithm in search, optimization, and machine learning. Addison: Wesley.

[13]. Hanzo, L., Yang, L.-L., Kuan, E.-L., \& Yen, K. (2003). Single- and multi-carrier DS-CDMA. NewYork: Wiley/IEEE Press.

[14] Sacchi, C., Donelli, M., D’Orazio, L., Fedrizzi, R.,\&De Natale, F. G. B. (2007). Genetic algorithmbased MMSE receiver for MC-CDMA systems transmitting over time-varying mobile channels. Electronics Letters, 43, 172-173.

[15] Lain, J.-K., \& Lai, J.-J. (2007). Ant colony optimisation-based multiuser detection for directsequence CDMA systems with diversity reception. IET Communications, 1, 556-561. DOI:10.1049/ietcom:20050456

[16]. Eberhart, R. C., \& Shi, Y. (2001). Tracking and optimizing dynamic systems with particle swarms. In Proceedings of IEEE Congress on Evolutionary Computation, Seoul, Korea (pp. 94-97). DOI:10.1109/CEC.2001.934376

[17]Liu, H., \& Li, J. (2008). A particle swarm optimization-based multiuser detection for receivediversityaided STBC systems. IEEE Signal Processing Letter, 15, 29-32. DOI:10.1109/LSP.2007.910315

[18]. Ratnaweera, A., Halgamuge, S. K., \& Watson, H. C. (2004). Self-organizing hierarchical particle swarm optimizer with time-varying acceleration coefficients. IEEE Transactions on Evolutionary Computation, 8 , 240-255. DOI:10.1109/TEVC.2004.826071

[19]. Soo, K. K., Siu, Y. M., Chan, W. S., Yang, L., \& Chen, R. S. (2007). Particle-swarm-optimization based multiuser detection for CDMA communication. IEEE Transactions on Vehicular Technology, 56,3006-3013. DOI:10.1109/TVT.2007.900383

[20]. Soo, S. H., \& Rao, S. S. (2000). Annealed neural network based multiuser detector in code division multiple access communications. IET Communnications, 47, 57-62. DOI: 10.1049/ipcom:20000228

[21] Fernando G. Lobol and Cl'audio F. Lima "Adaptive Population Sizing Schemes in Genetic Algorithms", Studies in Computational Intelligence (SCI), 185204 , 2007 DOI:10.1007/978-3-540-69432-8_9

[22] A. Gusmão and R. Dinis, "Iterative receiver techniques for cancellation of deliberate nonlinear distortion in OFDM-type transmission," in Proc. IEEE Int. OFDM Workshop, Dresden, Germany, Sep. 2004.

[23] Claudio Sacchi , Massimo Donelli and Francesco G. B. De Natale "Genetic-Algorithm-Assisted Maximum-Likelihood Detection of OFDM Symbols in the Presence of Nonlinear Distortions" IEEE Transactions On Communications, VOL. 55, NO. 5,
MAY 2007, pp.

DOI:10.1109/TCOMM.2007.896126

854-859.

[24] H. Ochiai, "Performance of optimal and suboptimal detection for uncoded OFDM systems with deliberate clipping and filtering," in Proc. IEEE GLOBECOM Conf., San Francisco, CA, Dec. 2003, vol. 3, pp. 1618-1622.

DOI:10.1109/TCOMM.2010.083110.09296

[25] Jiang, M., Ng, S.X., Hanzo, L., 2006. Hybrid iterative multiuser detection for channel coded space division multiple access OFDM systems. IEEE Trans. Veh. Technol., 55(1):115-127. [doi:10.1109/TVT.2005.861187].

[26] V. A. Valk'o "Self-calibrating Evolutionary Algorithms: Adaptive Population Size." Master's thesis, Free University Amsterdam, 2003.

[27] Yang, L.-L., \& Hanzo, L. (2003). Multicarrier CDMA systems a multiple access scheme for ubiquitous broadband wireless communication. IEEE Communications Magazine, 41, 116-124. DOI:10.1109/MCOM.2003.1235603

[28] Ho-Lung Hung · Jyh-HorngWen,"An Adaptive Multistage Multiuser Detector for MC-CDMA Communication Systems Using Evolutionary Computation Technique", vol 53, 2010, wireless personal communications, pp.613-633. DOI:10.1007/s11277-009-9722-3

[29]. Lee, S.-H., Hung, H.-L., Huang, Y.-F., \&Wen, J.-H. (2009). Performance analysis of PSO-based parallel interference canceller for MC-CDMA communication systems. European Transactions on Telecommunications,20(3), 287-297. DOI: 10.1002/ett.1313

[30]. Krusienski, D. J., \& Jenkins, W. K. (2005). Design and perform ance of adaptive systems based on structured stochastic optimization strategies. IEEE Circuits and Systems Magazine, 5(1), 8-20. DOI:10.1109/MCAS.2005.1405897

[31] H. Ochiai, "Performance of optimal and suboptimal detection for uncoded OFDM systems with deliberate clipping and filtering," in Proc. IEEE GLOBECOM Conf., San Francisco, CA, Dec. 2003, vol. 3, pp. 1618-1622. DOI:10.1109/GLOCOM.2003.1258511

[32] K.Seshadri Sastry, Prasad Babu M.S. (2012), "Non Data Aided SNR Estimation for OFDM Signals in Frequency Selective Fading Channels" Springer journal of Wireless Personal Communications, DOI 10.1007/s11277-012-0686-3, June 2012,. DOI: $10.1007 / \mathrm{s} 11277-012-0686-3$

[33] 2. *Seshadri Sastry.K, Prasad Babu .M.S (2012) "Secant Method Based ML Estimation of Carrier Frequency Offset in OFDM System”, International Journal of Computer Science and information Security, ISSN 1947-5500, April 2012, Vol 10(4), pp 125-128.

[34] K.Seshadri Sastry.K, Prasad Babu .M.S (2010), ” SNR Estimation for QAM Signals Using Fuzzy Logic Interface", IEEE ICCSIT 2010, July 2010, pp 413416 DOI:10.1109/ICCSIT.2010.5564920 
[35] C. Rapp, "Effects of HPA-nonlinearity on a 4DPSK/OFDM-signal for a digital sound broadcasting system," in Proc. 2nd Eur. Conf. Satellite Commun., Liege, Belgium, Oct. 1991, pp. 179-184.

[36]. K.Seshadri Sastry.K, Prasad Babu .M.S (2010)," AI Based Digital Companding Scheme for Software Defined Radio" IEEE ICSESS , 2010, pp 417- 419 DOI:10.1109/ICCSIT.2010.5563877

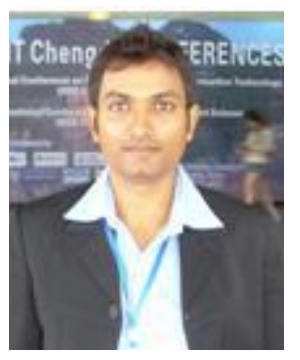

K.Seshadri Sastry was born in Srikakulam, Andhra Pradesh, India in 1978. He received B.E. degree in Electronics and Communications Engineering from Gulbarga University, India in 2001, M.Tech in VLSI Design from Bharath University, Chennai, India in 2005 . From 2001 to 2003 he worked as Assistant professor in SISTAM engineering collage, India and from 2005 to 2008 he worked as Associate professor in Chaitanya Engineering collage, Visakhapatnam, India. Since April 2008 he completed his Phd under the guidance of Prof.M.S.Prasad Babu, Department of Computer Science and Systems Engineering, Andhra University, Visakhapatnam, India. He published six research papers in International journals, attended and presented five research papers at three international conferences in India and China. $\mathrm{He}$ is presented working in Gandhi Institute of Engineering and Technology, Gunupur, Odisha as Associate Professor

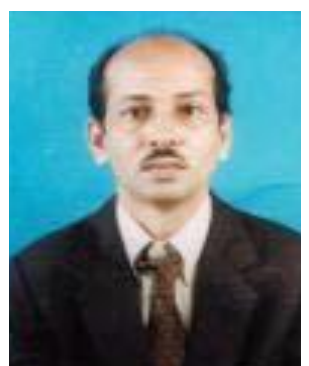

Prof. M.S.Prasad Babu was born on 12 - 08 - 1956 in Prakasam district of Andhra Pradesh, India. He obtained his B. Sc, M.Sc and M. Phil and Ph.D. degrees from Andhra University in 1976, 1978, 1981 and 1986 respectively. During his 28 years of experience in teaching and research, he attended about 28 National and International Conferences/ Seminars in India and contributed about 33 papers either in journals or in National and International conferences/ seminars. Prof. M.S. Prasad Babu has guided 98 student dissertations of B.E., B. Tech. M.Tech. \& Ph.Ds. Prof Babu presently working as senior Professor in the Department of Computer Science \& Systems Engineering of Andra University College of Engineering, Andhra University, Visakhapatnam 